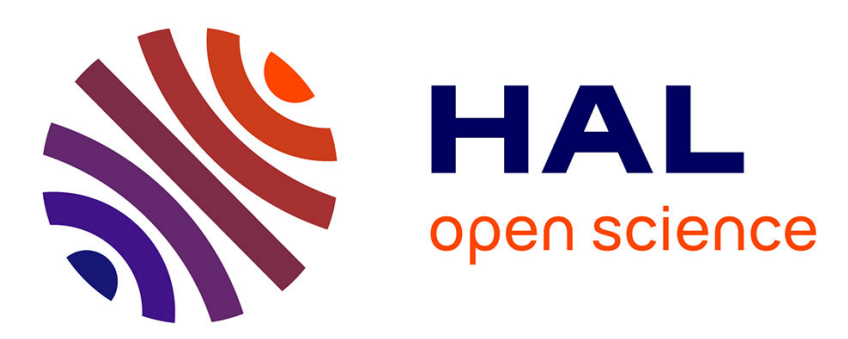

\title{
Laboratory spectroscopy of methoxymethanol in the millimeter-wave range
}

Roman Motiyenko, Laurent Margulès, D. Despois, Jean-Claude Guillemin

\section{To cite this version:}

Roman Motiyenko, Laurent Margulès, D. Despois, Jean-Claude Guillemin. Laboratory spectroscopy of methoxymethanol in the millimeter-wave range. Physical Chemistry Chemical Physics, 2018, 20 (8), pp.5509-5516. 10.1039/C7CP05932A . hal-01730207

\section{HAL Id: hal-01730207 https://hal.science/hal-01730207}

Submitted on 3 May 2018

HAL is a multi-disciplinary open access archive for the deposit and dissemination of scientific research documents, whether they are published or not. The documents may come from teaching and research institutions in France or abroad, or from public or private research centers.
L'archive ouverte pluridisciplinaire HAL, est destinée au dépôt et à la diffusion de documents scientifiques de niveau recherche, publiés ou non, émanant des établissements d'enseignement et de recherche français ou étrangers, des laboratoires publics ou privés. 


\section{Laboratory spectroscopy of methoxymethanol in the millimeter- wave range ${ }^{\dagger}$}

Roman A. Motiyenko, ${ }^{* a}$ Laurent Margulès ${ }^{a}$ Didier Despois,${ }^{b}$ and Jean-Claude Guillemin ${ }^{c}$

Methoxymethanol, $\mathrm{CH}_{3} \mathrm{OCH}_{2} \mathrm{OH}$ is a very interesting candidate for the detection in the interstellar medium since it can be formed by the recombination reaction between two radicals considered as intermediates in the methanol formation: $\mathrm{CH}_{3} \mathrm{O}$ (already detected in the ISM) and $\mathrm{CH}_{2} \mathrm{OH}$. It could be also formed by addition of $\mathrm{CH}_{3} \mathrm{O}$ on formaldehyde (another abundant compound in the ISM) followed by abstraction of a hydrogen radical. In this study, we present the first spectroscopic characterization of methoxymethanol in the millimeter-wave range augmented by high level quantum chemical calculations. The analysis revealed three stable conformations all exhibiting different large amplitude motions (LAM). For the analysis of the most stable conformation I we applied the model that accounts for hindered internal rotation of the methyl top. The analysis of the conformation III was performed taking the interaction between overall rotation and $\mathrm{OH}$ torsional motion into account. The conformation II was only tentatively assigned, as it exhibits several LAMs that significantly complicate theoretical description. Accurate spectroscopic parameters obtained in this study provide a reliable basis for the detection of methoxymethanol in the ISM.

\section{Introduction}

To select new target compounds for the interstellar medium (ISM), the most usual approach is probably the analogy with the compounds already detected in this medium. ${ }^{\ddagger}$ However, considering some plausible chemical reactions between the detected compounds can lead to more original targets. These approaches are strongly supported when such species have been found in lab simulations of the chemistry of the ISM in the gas phase or on the grains.

Methanol has been detected a long time ago in the ISM. ${ }^{1}$ Its formation in this medium probably occurs on the grains ${ }^{2,3}$ by the reaction of hydrogen radicals on solid carbon monoxide leading to methanol via the formaldehyde. ${ }^{4,5}$ The reaction of hydrogen on formaldehyde can give two products, the hydroxymethyl $\mathrm{CH}_{2} \mathrm{OH}$ and methoxy $\mathrm{CH}_{3} \mathrm{O}$ radicals but the former can also be produced by reaction of methanol on hydrogen radical. The methoxy radical has been recently detected in the ISM ${ }^{6}$ while the study of the millimeter spectrum of the hydroxymethyl radical has been only recently performed but was not followed by its detection in the ISM up to date. ${ }^{7}$ In the case where it is formed in this medium, a possible explanation could be a rapid radical recombination in the grains to form a dimer prior to the desorption of the monomer. ${ }^{5}$ The dimer, ethyleneglycol, has already been observed in the ISM. ${ }^{8}$ If this hypothesis is correct and because the methoxy radical has been unambiguously detected, the recombination of both radicals $\left(\mathrm{CH}_{3} \mathrm{O}+\mathrm{CH}_{2} \mathrm{OH}\right)$ could lead to the formation of another product, the methoxymethanol $\mathrm{CH}_{3} \mathrm{OCH}_{2} \mathrm{OH}$. Note that the association of two $\mathrm{CH}_{2} \mathrm{OH}$ radicals to form $\left(\mathrm{CH}_{2} \mathrm{OH}\right)_{2}$, although appealing by its simplicity, seems to be not favoured as the $\mathrm{CH}_{2} \mathrm{OH}$ radical has a low mobility due to $\mathrm{OH}$ bonds. ${ }^{9}$ Methoxymethanol could also be produced by addition of $\mathrm{CH}_{3} \mathrm{O}$ radical on formaldehyde followed by hydrogen abstraction if such reactions are barrier-less:

$$
\begin{gathered}
\mathrm{CH}_{3} \mathrm{O}+\mathrm{H}_{2} \mathrm{CO} \longrightarrow \mathrm{CH}_{3} \mathrm{OCH}_{2} \mathrm{O} \\
\mathrm{CH}_{3} \mathrm{OCH}_{2} \mathrm{O}+\mathrm{H} \longrightarrow \mathrm{CH}_{3} \mathrm{OCH}_{2} \mathrm{OH}
\end{gathered}
$$

At the opposite of the ethylene glycol isomer, few studies have focused on methoxymethanol. Nevertheless, it has been demonstrated that low-energy (1-20 eV) electron-induced reactions in condensed thin films of methanol and post-irradiation temperature-programmed desorption of $\mathrm{CH}_{2} \mathrm{OH}$ and $\mathrm{CH}_{3} \mathrm{O}$ produce ethylene glycol and methoxymethanol. ${ }^{10,11}$ Such reactions could play a dominant role in ionizing radiation-induced chemical syn-

\footnotetext{
a Laboratoire de Physique des Lasers, Atomes et Molécules, UMR CNRS 8523 - Université de Lille 1 Sciences et Technologies,

Villeneuve d'Ascq Cedex, France.E-mail: roman.motienko@univ-lille1.fr

${ }^{b}$ Laboratoire d'Astrophysique de Bordeaux, Université de Bordeaux, CNRS, Pessac, France.

${ }^{c}$ Institut des Sciences Chimiques de Rennes, UMR 6226 CNRS - ENSCR, Rennes, France.

$\dagger$ Electronic Supplementary Information (ESI) available: spectral assignment and fits. See DOI: 10.1039/C7CP05932A/

$\ddagger$ http://www.astro.uni-koeln.de/cdms/molecules
} 


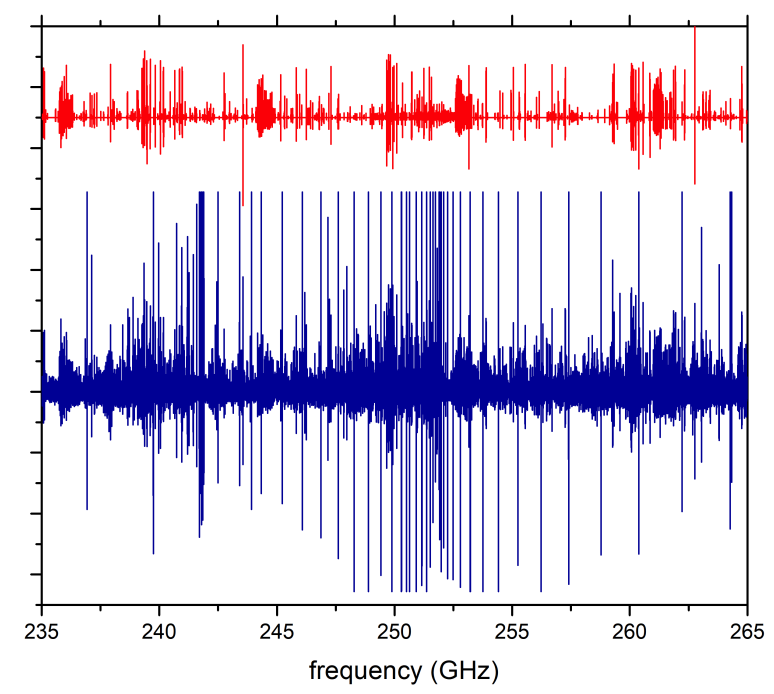

Fig. $1 \mathrm{In}$ blue: the recorded spectrum between 235 and $265 \mathrm{GHz}$ dominated by $Q$-type $K_{a}=3 \leftarrow 2$ transitions of methanol. The methanol lines were saturated at analog-to-digital converter to obtain higher dynamic range for weaker lines of methoxymethanol. In red the spectra of conformers I and III of methoxymethanol simulated on the basis of the results obtained in this study. The amplitude of the synthetic spectra is scaled on assigned lines of the methoxymethanol.

thesis of the ISM and, it should be noted that most of the products identified in these studies starting from methanol have been detected in this medium. ${ }^{12}$ In addition, in these studies it was suggested that $\mathrm{CH}_{3} \mathrm{OCH}_{2} \mathrm{OH}$ could serve as a tracer molecule for electron-induced reactions in the interstellar medium. ${ }^{13}$ Consequently, on the basis of all these observations, we have considered methoxymethanol as a good candidate for the ISM since its detection or non-detection would improve the current knowledge in the interstellar chemistry.

The detection of new compounds in the ISM needs to have in hand the spectra of the target compound for comparison with spectra coming from interstellar clouds. For over more than sixty years, rotational spectroscopy has allowed the detection of many species in the ISM but only compounds with a permanent dipolar moment can be concerned. The rotational spectrum of methoxymethanol has never been studied before. Previous publications include high-level theoretical calculations that yielded rotational constants, harmonic force field, and the information on the conformational stability and some transitions states. ${ }^{14}$ These calculations were performed in view of subsequent experimental observations of the rotational spectrum of methoxymethanol via $O\left({ }^{1} \mathrm{D}\right)$ insertion reaction with dimethyl ether since such reaction was successfully applied for methane and ethylene to produce respectively methanol and vinyl alcohol. ${ }^{15}$ In the present study, we report the first assignment and analysis of the rotational spectrum of methoxymethanol that was synthesized via chemical approach described below. We also report the results of quantum chemical calculations performed to aid in the interpretation of the observed spectra.

\section{Experiment}

\section{Synthesis}

The synthesis of Priede et al. ${ }^{16}$ has been modified to obtain methoxymethanol on a preparative scale. Paraformaldehyde ( $6.60 \mathrm{~g}$, equivalent to $0.22 \mathrm{~mol}$ of formaldehyde) and anhydrous methanol (128 g, $4.0 \mathrm{~mol})$ were introduced in a $250 \mathrm{~mL}$ cell equipped with a stirring bar and a stopcock. The suspension was cooled at $77 \mathrm{~K}$ and the cell degassed before to close the stopcock. The suspension was heated at $95^{\circ} \mathrm{C}$ for $3 \mathrm{~h}$, whereupon clear solution formed. After cooling at room temperature, the methoxymethanol was purified by trap-to-trap distillation in vacuo $(0.1 \mathrm{mbar})$. Three slow distillations with partially selective condensation in a trap immersed in a bath cooled at $-55^{\circ} \mathrm{C}$ led to methoxymethanol containing less than $5 \%$ of methanol. Yield: $7.6 \mathrm{~g}(56 \%)$. ${ }^{1} \mathrm{H} \mathrm{NMR}$ $\left(\mathrm{CDCl}_{3}\right) \delta 3.37\left(\mathrm{~s}, 3 \mathrm{H}, \mathrm{CH}_{3}\right) ; 4.38\left(\mathrm{t}, 1 \mathrm{H},{ }^{3} \mathrm{~J}_{\mathrm{HH}}=7.9 \mathrm{~Hz}, \mathrm{OH}\right) ; 4.67\left(\mathrm{~d}, 2 \mathrm{H},{ }^{3} \mathrm{~J}_{\mathrm{HH}}=7.9 \mathrm{~Hz}, \mathrm{CH}_{2}\right) .{ }^{13} \mathrm{C} \mathrm{NMR}$ $\left(\mathrm{CDCl}_{3}\right) \delta 55.1\left({ }^{1} \mathrm{~J}_{\mathrm{HH}}=142.3 \mathrm{~Hz}(\mathrm{q}), \mathrm{CH}_{3}\right) ; 90.7\left({ }^{1} \mathrm{~J}_{\mathrm{HH}}=162.1 \mathrm{~Hz}(\mathrm{t}), \mathrm{CH}_{2}\right)$. 


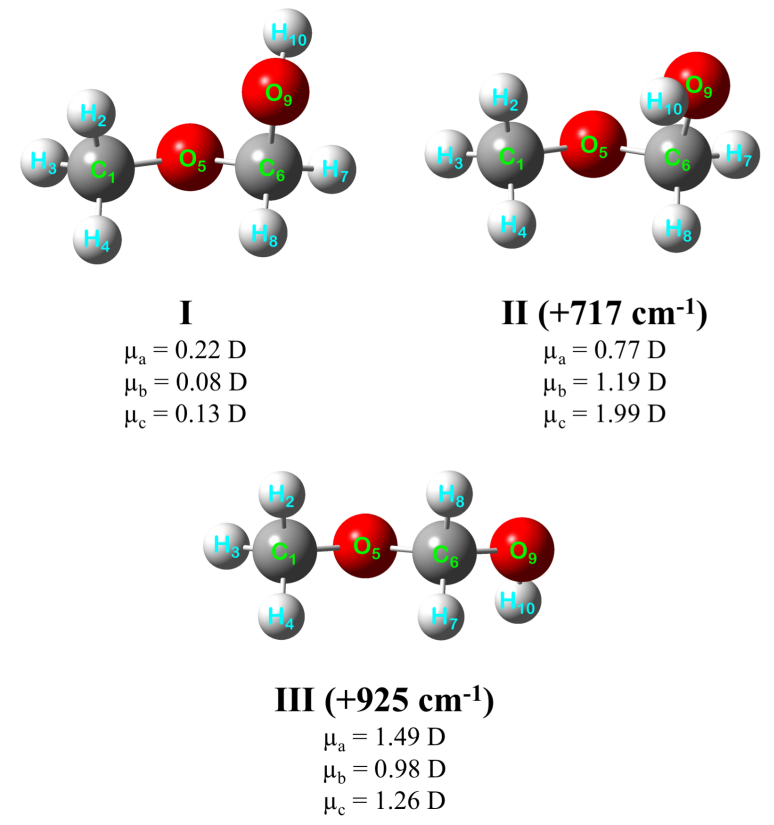

Fig. 2 Structure, atom numbering, relative energies, and dipole moment components of three stable conformations of methoxymethanol. The energies and dipole moments are obtained from MP2/aug-cc-pVTZ calculations.

\section{Conventional Absorption Spectroscopy}

We recorded the rotational spectrum of methoxymethanol using fast-scan terahertz spectrometer in Lille. The details of the spectrometer except of the fast-scan feature are described by Zakharenko et al. ${ }^{17}$. As a radiation source in the spectrometer, we use commercially available VDI frequency multiplication chain that is driven by home-made fast sweep frequency synthesizer. The fast sweep system is based on the up-conversion of AD9915 direct digital synthesizer (DDS) operating between 320 and $420 \mathrm{MHz}$ into $\mathrm{Ku}$ band by mixing the signals from AD9915 and Agilent E8257 synthesizers with subsequent sideband filtering. The DDS provides rapid frequency scan with up to $50 \mu \mathrm{s} /$ point frequency switching rate. In the present study, the spectrum was recorded in the frequency ranges $150-200 \mathrm{GHz}, 220-330 \mathrm{GHz}$ and $400-460 \mathrm{GHz}$. Because of the methoxymethanol line weakness the spectrum was scanned with slower rate of $1 \mathrm{~ms} /$ point, and to additionally increase the signalto-noise ratio, each spectrum was averaged 8 times. Owing to kinetic instability of methoxymethanol the measurements were performed in the so-called "flow mode". During the experiment, the absorption cell, which consisted of $1.2 \mathrm{~m}$ Pyrex glass tube with Teflon windows, was kept at room temperature, whereas the sample of methoxymethanol was cooled at a temperature of about $-50^{\circ} \mathrm{C}$ and evaporated outside of the cell. Then, the sample was continuously injected through a side opening at one end of the cell and pumped out through another side opening at the other end. The optimum gas pressure in the cell was kept close to $25 \mathrm{~Pa}$. The recorded spectra are very dense mostly owing to same order of magnitude intensities of the rotational lines of all three stable conformations of methoxymethanol as described below. The spectra are also dominated by the lines of methanol and formaldehyde that may originate either from impurities in the sample or from decomposition products. An example of the recorded spectrum between 235 and $265 \mathrm{GHz}$ is shown on Fig 1. To obtain higher dynamic range with respect to weak lines of methoxymethanol, we saturated the intensities of strong lines in the analog-to-digital converter at the detection. Therefore, the strongest lines presented on Fig. 1 are cut off and do not follow characteristic at $2 f$ detection over/below baseline intensities ratio of 3/1. All the methanol and formaldehyde lines were excluded from the spectrum, as they hampered the analysis owing to their saturated intensities.

\section{Theoretical Calculations}

The present quantum chemical calculations were performed using the Gaussian 09 suite of programs. ${ }^{18}$

First, we performed structural optimization and harmonic force field calculations at MP2 ${ }^{19}$ level of theory and using Peterson and Dunning's correlation-consistent aug-cc-pVTZ basis set, which is of triple- $\zeta$ quality 


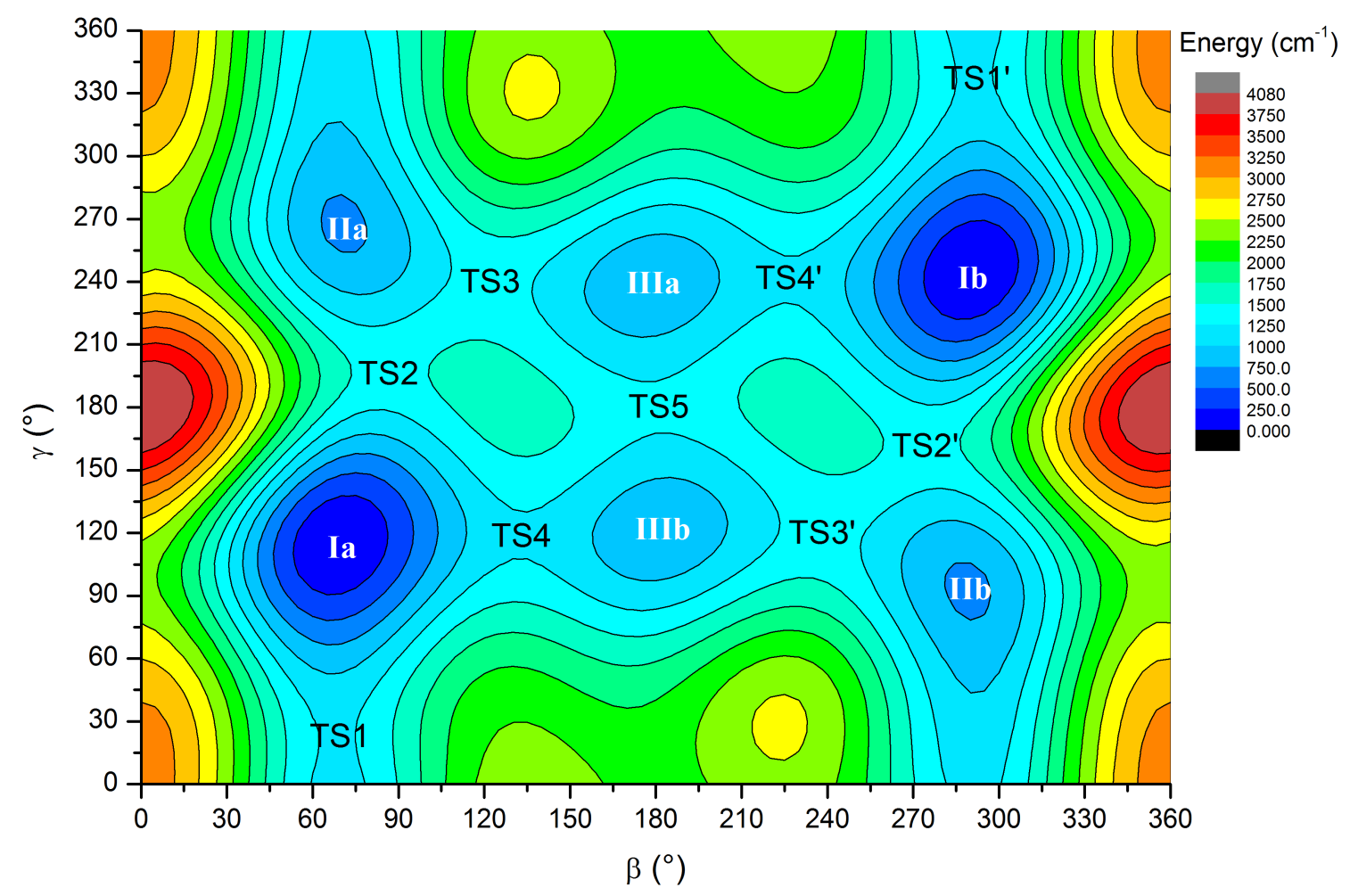

Fig. 3 The calculated potential energy surface of methoxymethanol as a function of two dihedral angles $\beta($ O9-C6-O5-C1) and $\gamma(\mathrm{H} 10-\mathrm{O} 9-\mathrm{C} 6-\mathrm{O} 5)$. See Fig. 2 for the definition of the coordinates.

Table 1 The energies of transition states of methoxymethanol with respect to the conformer I. The transition states are defined on Fig. 3

\begin{tabular}{cccc}
\hline $\begin{array}{c}\text { Transition } \\
\text { state }\end{array}$ & $\beta\left(^{\circ}\right)$ & $\gamma\left(^{\circ}\right)$ & $\begin{array}{c}\text { Relative } \\
\text { energy }\left(\mathrm{cm}^{-1}\right)\end{array}$ \\
\hline TS1 & 66.2 & 21.5 & 1256 \\
TS2 & 82.3 & 198.5 & 1468 \\
TS3 & 122.7 & 238.4 & 1531 \\
TS4 & 132.2 & 119.5 & 1427 \\
TS5 & 180 & 180 & 1416 \\
\hline
\end{tabular}

and includes both diffuse and polarized functions. ${ }^{20}$ The geometries were fully optimized using the "tight" convergence option. As it was found in the previous study, methoxymethanol has three stable conformations denoted here as I, II and III. Their structures, atom numbering, and calculated dipole moment values are shown on Fig. 2. The conformer I is the most stable one. The relative energies of the conformers II and III resulting from structural optimization are respectively 717 and $925 \mathrm{~cm}^{-1}$. The relative energies with zero-point energy correction from harmonic force field calculations are correspondingly 672 and $813 \mathrm{~cm}^{-1}$. Two most stable conformations I and II have molecular skeleton formed by heavy atoms in gauche configuration, and they differ only by the orientation of the hydroxyl group hydrogen. Consequently, conformations I and II have rather similar rotational constants (see Table 3) but quite different components of the permanent dipole moment. The conformation III is characterized by planar heavy atoms skeleton, and by gauche configuration of the $\mathrm{OH}$ group.

Second, we calculated two-dimensional potential energy surface (PES) of methoxymethanol. Owing to large computational volume, the density functional theory (DFT) calculations were performed with Becke's threeparameter hybrid functional ${ }^{21}$ employing the Lee, Yang, and Parr ${ }^{22}$ correlation functional (B3LYP), and using $6-311++\mathrm{G}(3 \mathrm{df}, 2 \mathrm{pd})$ basis set. In methoxymethanol, the heavy atoms connected by three single bonds are the origins of three large amplitude motions (LAM). The LAMs may be parametrized by three dihedral angles: $\alpha$ (C6-O5-C1-H3, methyl torsion), $\beta$ (O9-C6-O5-C1, skeletal torsion) and $\gamma(\mathrm{H} 10-\mathrm{O} 9-\mathrm{C} 6-\mathrm{O} 5$, OH torsion). For each conformation, the methyl torsion may be described by typical three equivalent minima on one-dimensional PES which is approximated using the following expansion formula: 
Table 2 Parameters of the potential function $V(\alpha)$ (in $\mathrm{cm}^{-1}$, Eq. 1) describing the methyl group torsion for three stable conformations of methoxymethanol determined by quantum chemical calculations

\begin{tabular}{lccc}
\hline \multicolumn{2}{c}{ Conformer Parameter } & Value $^{\mathrm{a}}$ & Value $^{\mathrm{b}}$ \\
\hline \multirow{2}{*}{$\mathrm{I}$} & $V_{3}$ & $567.3(34)$ & $552.6(42)$ \\
& $V_{6}$ & $-16.2(30)$ & $-14.8(37)$ \\
II & $V_{3}$ & $708.2(38)$ & $699.4(35)$ \\
& $V_{6}$ & $-6.5(21)$ & $-4.3(12)$ \\
III & $V_{3}$ & $798.8(24)$ & $772.7(29)$ \\
& $V_{6}$ & $-15.6(21)$ & $-17.4(26)$ \\
\hline
\end{tabular}

${ }^{\text {a }} \mathrm{MP} 2$ /aug-cc-pVTZ.

b $\mathrm{M} 06-2 \mathrm{X} / 6-311++\mathrm{G}(3 \mathrm{df}, 3 \mathrm{pd})$.

$$
V(\alpha)=\frac{V_{3}}{2}(1-\cos 3 \alpha)+\frac{V_{6}}{2}(1-\cos 6 \alpha)+\ldots
$$

In the previous study, ${ }^{14}$ the barrier to internal rotation of the methyl top for conformation I was estimated to be $669 \mathrm{~cm}^{-1}$. The other two LAMs are two-dimensional problems as two bond angles need to be changed along the pathway between equivalent minima. We calculated the PES as function of two dihedral angles and that is presented on Fig. 3. The calculations were performed by varying angles $\beta$ and $\gamma$ in the range from $0^{\circ}$ to $360^{\circ}$ by a step of $5^{\circ}$. The geometry was fully optimized at each step. Consequently, the energy of each point on PES corresponds to the optimized structure. As it may be seen from Fig. 3, each conformation is characterized by two energetically equivalent local minima on PES denoted with letters $a$ and $b$. The minima are connected by pathways across five different transitions states TS1 to TS5. Owing to symmetry properties, the transition states from TS1 to TS4 have two equivalent configurations. The information on different barrier heights may be obtained directly from the calculated PES. To provide more precision and a point of comparison with the calculations of stable conformers, the transition states were further optimized using "tight" convergence criteria at MP2 level and with aug-cc-pVTZ basis set. As expected, each optimization produced one negative vibrational frequency that corresponds to the first order saddle point. The energies of all five transitions states with respect to the conformer I are presented in Table 1. From these optimizations, the most interesting result is obtained for TS5 that separates two equivalent configurations of the conformer III. Taking the relative energy of the conformer III into account, the barrier to interconversion between two gauche configurations of the conformer III is about $492 \mathrm{~cm}^{-1}$. Consequently, tunneling splittings owing to $\mathrm{OH}$ torsional motion are expected in the rotational spectrum of the conformer III. The splittings owing to tunneling between two equivalent configurations of conformers I, and II are also possible. However, higher barrier heights and also much larger barrier widths should make tunneling probabilities and thus tunneling splittings much smaller compared to those of conformer III.

Finally, the barriers to internal rotation of the methyl group were calculated for all three conformations. In these calculations, we retained MP2/aug-cc-pVTZ as well as DFT M06-2X/6-311++G(3df, 3pd) method/basis set combinations. ${ }^{23}$ The latter method has previously shown to provide relatively high precision of the results as compared to experimental values ${ }^{24,25}$ demanding at the same time much less computational effort. In the present case, the computational time required for calculations was smaller by a factor of seven for M06-2X/6$311++\mathrm{G}(3 \mathrm{df}, 3 \mathrm{pd})$ than for MP2/aug-cc-pVTZ. For each conformer, to estimate the height of the barrier to methyl torsion, we calculated one-dimensional PES by varying the angle alpha from $0^{\circ}$ to $360^{\circ}$ by a step of $5^{\circ}$. Then, the resultant PES was fitted to Eq.1 to get the values of the $V_{3}$ and $V_{6}$ terms. The results of the fits are presented in Table 2. For the conformation I, the comparison of the theoretical values of $V_{3}$ with the experimental one is provided in the 'Analysis' section, and in Table 3.

The values of the barrier heights for all three conformations are influenced mostly by steric effects. In this view, the conformation III may be compared to dimethyl ether, $\mathrm{CH}_{3} \mathrm{OCH}_{3},{ }^{26}$ in which the main contribution to relatively high barrier of $925 \mathrm{~cm}^{-1}$ is due to the steric interaction between two pairs of out-of-plane hydrogen atoms. For the conformations I and II, the interaction is reduced as one of the hydrogens is replaced by hydroxyl group oxygen. In the absence of steric repulsion between hydrogen atoms, the barrier to internal rotation is determined by other effects. Here one may give examples of the $V_{3}$ value of $372 \mathrm{~cm}^{-1}$ for methyl formate, $\mathrm{CH}_{3} \mathrm{OCHO},{ }^{27}$ or $399 \mathrm{~cm}^{-1}$ for methyl cyanate, $\mathrm{CH}_{3} \mathrm{OCN} .{ }^{28}$ In case of the conformer I, the interaction between a pair of hydrogen atoms increases the barrier height, as compared to the two examples cited above. The 
Table 3 Rotational and torsional parameters of conformer I of methoxymethanol

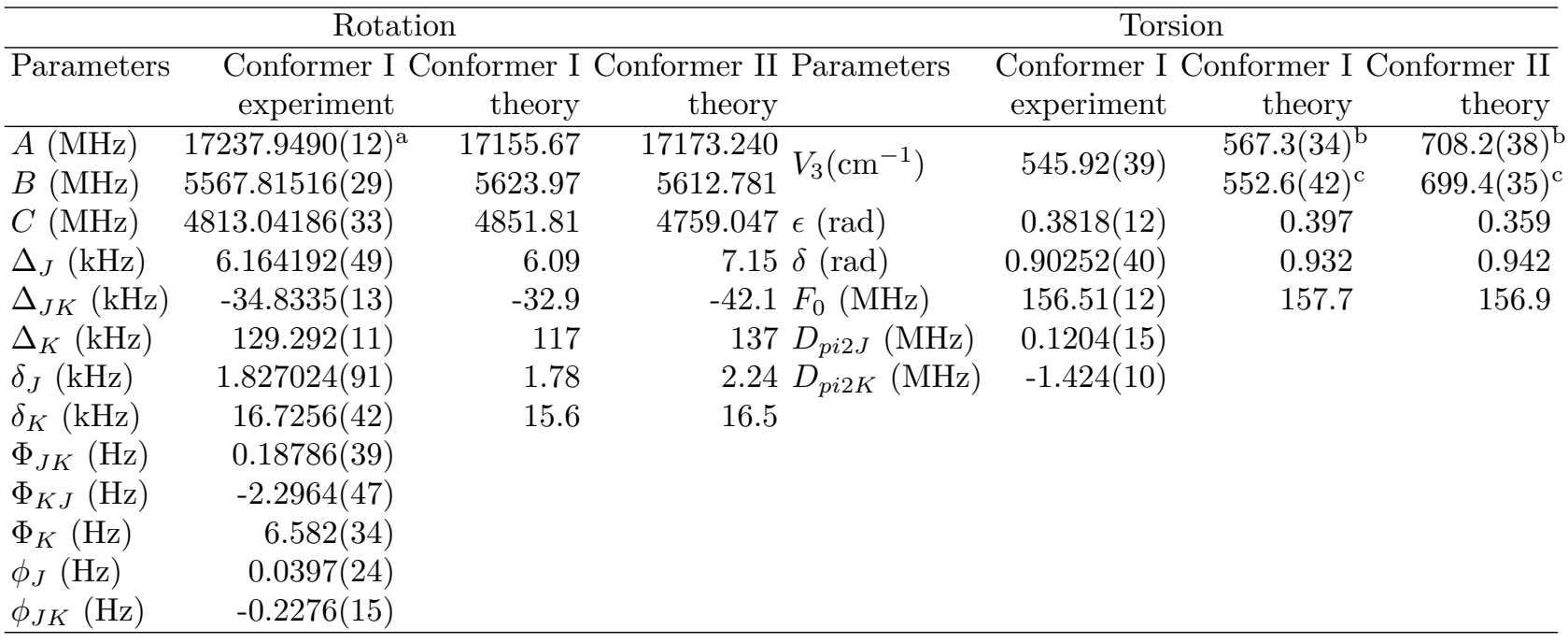

a Numbers in parentheses are two standard deviations in the same units as the last digit.

b MP2/aug-cc-pVTZ calculations.

c $\mathrm{M} 06-2 \mathrm{X} / 6-311++\mathrm{G}(3 \mathrm{df}, 3 \mathrm{pd})$ calculations.

conformer II represents an intermediate case between I and III, where in addition to the interaction between a pair of hydrogens, an interaction between hydrogens of methyl and hydroxy groups may take place leading to higher barrier to internal rotation in comparison with conformer I.

\section{Analysis}

According to ab initio calculations, the most stable conformer of methoxymethanol has the lowest dipole moment, while two others conformations are significantly less stable, but they have much higher dipole moments. By combining Boltzmann factors at room temperature and line strength proportional to dipole moment squared one gets a situation where the intensities of $a$-type transitions of all three conformations are comparable. Our calculations show that normalized relative intensities of a-type transitions of three conformations are I:II:III = 0.51:0.20:0.29. The experimental spectrum of methoxymethanol qualitatively confirms this estimation.

Conformer I. The conformer I was assigned on the basis of the spectral predictions obtained using the results of quantum chemical calculations. To account for the internal rotation of the methyl top, we used the Hamiltonian model based on combined internal and principal axes approaches which is realized in the XIAM code. $^{29}$ In XIAM, pure rotational part of the Hamiltonian operator is defined in the principal axis system, whereas the part that describes the internal rotation is defined in the internal axis system chosen to reduce the effect of the angular momentum generated by the torsional motion. After the diagonalization of the torsional part of the Hamiltonian, the resulting eigenvalues are rotated (transformed) to the principal axis system, and a global solution of the torsion-rotation problem is thus obtained. The advantage of XIAM code is that the main parameters of the Hamiltonian are directly linked to molecular structure. In addition to usual rotational constants, the rotation of the internal axis system with respect to the principal axis system is defined via two angles $\epsilon$ and $\delta$ that may be adjusted in fitting of experimental data. Both angles describe the orientation of the methyl top, $\delta$ is the angle between the internal rotation axis and the principal axis $z$, and $\epsilon$ is the angle between the projection of the internal rotation axis onto $x y$-plane and the principal axis $x$. Another important adjustable parameter $F_{0}$ is the inverse of the inertia moment of the methyl top.

The initial predictions of the rotational spectrum of the conformation I were calculated using XIAM code with rotational and centrifugal distortion constants as well as $\delta, \epsilon, V_{3}$ and $F_{0}$ parameters resulted from optimized ab initio molecular structure and harmonic force field. Such calculations were very important, as the initial assignment of the conformation I lines in very dense spectrum was a difficult task. Indeed, due to extremely crowded spectra we were not able to recognize typical high- $K_{a}$ patterns of ${ }^{a} R_{0,1}$ series transitions that were predicted to have the highest intensity in the frequency range of the measurements. Instead of searching for such patterns, we focused on low- $K_{a}$ transitions that were finally assigned owing to particular multiplet 


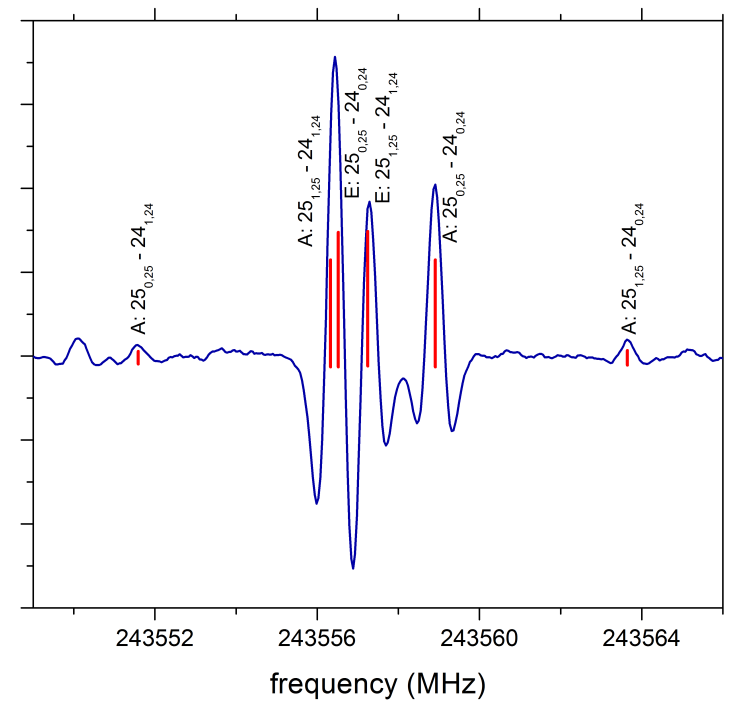

Fig. 4 Example of low- $K_{a} A-E$ symmetry multiplet due to internal rotation splittings in the conformation I of methoxymethanol. Relative intensities of individual components are indicated by red sticks.

structure produced by torsional splittings. Typically, the tunneling through the torsional barrier splits each rotational transition into two components of equal intensity labeled according to symmetry species $A$ and $E$ of $C_{3 v}$ symmetry point group that describes the methyl top. In case of strong torsion-rotational interaction, the relative intensities of the components may vary, and additional splittings may arise producing thus more complicated pattern than simple doublet. According to initial predictions, such interactions occur for low- $K_{a}$ transitions. An example of such multiplet is shown on Fig. 4. In the spectra, we found multiplets similar to predicted ones but shifted in frequency by several hundreds of megahertz. The first assignment and fitting of such multiplets to the Hamiltonian model used in XIAM code allowed the determination of several parameters and significantly reduced the shift between predicted and observed multiplets. The following assignment was performed in a usual "bootstrap" manner, when the results of the least-squares fit of transitions with lower values of quantum numbers were used to calculate the predictions of transitions with higher values of quantum numbers.

In total, we assigned 1176 rotational transitions of the conformer I in the ground vibrational state. All the transitions were fitted to the model containing 19 parameters with overall root-mean-square deviation of $0.04 \mathrm{MHz}$. The fitted parameters are presented in Table 3 and compared to the results of ab initio calculations. One may notice generally good agreement between theoretical calculations and experimental values. In Table 3 , the rotational constants of the conformer II are also given. As it may be seen, the rotational constants of the two most stable conformations are rather close, and simple comparison between theoretical and experimental values is not enough to determine which conformation was assigned and analyzed. To distinguish between conformations, we used dipole moment components. Theoretical calculations show that for the conformation $\mathrm{I}$, the $a$-type transitions should be much stronger than $b$-type, as $\mu_{a}$ dipole moment component is at least two times higher that $\mu_{b}$. Whereas for the conformation II, both the $a$ - and $b$-type transitions should have approximately the same intensities. The multiplet presented on Fig. 4 is composed by strong $a$-type transitions and weak $b$-type transitions, and consequently, it may be attributed to the conformation I.

The determined value of the barrier to internal rotation compares well with the values obtained by two different calculations presented above. The $V_{3}$ value may also be used as additional evidence of the conformer I assignment. Taking previous results into account, one may validate the choice of DFT M06-2X method as a lower computational cost alternative for the evaluation of torsional barrier heights. Based on the present analysis of the conformer I, reliable frequency predictions of the rotational spectrum in the ground vibrational state are obtained in the frequency range up to $500 \mathrm{GHz}$ and for all transitions involving quantum numbers $0<J<55$ and $0<K_{a}<20$.

Conformer II. The rotational constants of the conformer II listed in Table 3 were estimated from MP2/augcc-pVTZ calculations by applying scaling coefficients. The latter were obtained via direct comparison between 
theoretical and experimental constants of the conformer I. The spectral predictions calculated using scaled rotational constants of the conformer II allowed the assignment of a series of $K_{a}=0$ and $K_{a}=1{ }^{a} R_{0,1}$ transitions. However, the following assignment process was difficult as the assigned lines could not be fitted within experimental accuracy and using newly calculated prediction one could hardly assign higher $K_{a}$ lines. The fit produced unreasonably high values of centrifugal distortion constants that deviated rather significantly from those predicted from quantum chemical calculations. In this way, we were able to assign the transitions up to $K_{a}=2$. We were also able to assign the origins of high- $K_{a}{ }^{c} Q$ and ${ }^{c} R$ type bands, as the $\mu_{c}$ dipole moment component is the highest for the conformer II. However, compared to calculated predictions ${ }^{c} Q$ series contained much more lines indicating some additional splittings of rotational lines. The problem of centrifugal distortion constants may be explained by quite asymmetric character of the potential well of the conformer II (see Fig. 2) that deviates significantly from harmonic oscillator approximation in which the theoretical constants were obtained. The problem of additional splittings may arise from coupling between methyl torsion and two other LAMs. Unfortunately, at present, there is no model that is able to treat the problem of methyl top internal rotation and two tunneling motions within the accuracy of high-resolution terahertz experiment. Therefore, we provide only the list of assigned lines of the conformer II in the $\mathrm{ESI}^{\dagger}$.

Conformer III. The conformer III is a prolate asymmetric top close to symmetric top limit $\kappa=0.98$. Owing to this feature, the high $K_{a}{ }^{a} R_{0,1}$ bands represent a congested series of lines spaced on frequency scale approximately by $B+C$ and thus easily assignable in broadband spectra. Close inspection of each series revealed that each transition in series has a double structure. The doublet splittings originate from tunneling between two equivalent gauche configurations of the conformer III. The tunneling splits each rotational energy level into two components usually labeled as $0^{-}$and $0^{+}$. To treat the tunneling splittings, we applied the method based on reduced-axis-system (RAS) approach proposed by Pickett. ${ }^{30}$ It is well suited for molecules with a double minimum-potential. In matrix form, in the basis of individual wavefunctions of each tunneling substate $\left|0^{+}\right\rangle$ and $\left|0^{-}\right\rangle$, the RAS Hamiltonian has the following form:

$$
H=\left(\begin{array}{cc}
H_{\mathrm{rot}}-H_{\Delta} & H_{\mathrm{I}} \\
H_{\mathrm{I}} & H_{\mathrm{rot}}+H_{\Delta}
\end{array}\right)
$$

In Eq.2, $H_{\text {rot }}$ is the standard Watson S-reduction Hamiltonian in the $I^{r}$ coordinate representation, $H_{\Delta}$ is the part of the Hamiltonian that allows fitting averaged rotational constants for both tunneling substates:

$$
H_{\Delta}=E^{*}+E_{J}^{*} P^{2}+E_{K}^{*} P_{z}^{2}+E_{2}^{*}\left(P_{+}^{2}+P_{-}^{2}\right)+\ldots
$$

with the energy difference between two substates $\Delta E=2 E^{*} . H_{\mathrm{I}}$ is a perturbation Hamiltonian containing $F_{x y}\left(P_{x} P_{y}+P_{y} P_{x}\right)$, and $F_{y z}\left(P_{y} P_{z}+P_{z} P_{y}\right)$, terms and their centrifugal distortion corrections. The non-diagonal terms determine the orientation of the reduced axis system with respect to the principal axis system.

In total, we assigned more than 700 rotational transitions of the conformer III. All the assigned transitions are of $a$-type. Owing to structural features, the parameter $\rho$ that describes the coupling between internal rotation of the methyl top and overall rotation of the molecule is much higher for the conformer III $\left(\rho_{\text {III }}=0.19\right)$ compared to two other conformers $\left(\rho_{\mathrm{I}, \mathrm{II}}=0.07\right)$. For the conformer III, in combination with relatively high barrier to internal rotation, it leads to unresolvable tunneling splittings for the $a$-type transitions and rather large splittings of 1 to $15 \mathrm{MHz}$ for $b$ - and $c$-type transitions. This fact may explain why we were able to assign only $a$-type transitions of conformer the III, as the model of the RAS Hamiltonian does not take the methyl top internal rotation into account. In addition, the $c$-type transitions connect two tunneling substates $\left|0^{+}\right\rangle$and $\left|0^{-}\right\rangle$and, therefore, their frequencies strongly depend on $\Delta E$ parameter.

The assigned transitions frequencies were fitted within experimental accuracy using the Hamiltonian in Eq.2 with overall rms deviation of $0.048 \mathrm{MHz}$. The results of the fit are presented in Table 4 . The energy difference between two tunneling substates follows the general tendency observed for similar molecules exhibiting $\mathrm{OH}$ torsion. In the case of 2-hydroxyacetonitrile $\mathrm{HOCH}_{2} \mathrm{CN},{ }^{31}$ theoretical calculations yield the barrier height of $427 \mathrm{~cm}^{-1}$, while the energy difference $\Delta E$ determined from the rotational spectra analysis is $3.76 \mathrm{~cm}^{-1}$. For a similar molecule, 4-hydroxy-2-butynenitrile $\mathrm{HOCH}_{2} \mathrm{C}_{3} \mathrm{~N},{ }^{32}$ theoretically estimated barrier height of $365 \mathrm{~cm}^{-1} \mathrm{corre}^{-}$ sponds to the observed tunneling splitting of $4.55 \mathrm{~cm}^{-1}$. In the present study, the barrier to $\mathrm{OH}$ torsion is predicted to be higher than in two previous cases, $492 \mathrm{~cm}^{-1}$, which is consistent with the $\Delta E$ value from the least-squares fit of $3.02 \mathrm{~cm}^{-1}$. 
Table 4 Rotational parameters of the conformer III of methoxymethanol

\begin{tabular}{|c|c|c|}
\hline Parameters & Experiment & theory $^{\mathrm{a}}$ \\
\hline$A(\mathrm{MHz})$ & \multicolumn{2}{|c|}{$32328.14(10)^{\mathrm{b}} 32534.76$} \\
\hline$B(\mathrm{MHz})$ & $4350.32898(47)$ & 4366.71 \\
\hline$C(\mathrm{MHz})$ & $4070.97615(46)$ & 4086.61 \\
\hline$D_{J}(\mathrm{kHz})$ & $1.073687(51)$ & 1.07 \\
\hline$D_{J K}(\mathrm{kHz})$ & $-3.3717(26)$ & -3.46 \\
\hline$D_{K}(\mathrm{kHz})$ & {$[121.2]^{\mathrm{c}}$} & 121.2 \\
\hline$d_{1}(\mathrm{kHz})$ & $-0.10047(11)$ & -0.102 \\
\hline$d_{2}(\mathrm{kHz})$ & $-0.001644(55)$ & -0.00128 \\
\hline$H_{K J}(\mathrm{~Hz})$ & $-0.406(15)$ & \\
\hline$\Delta E(\mathrm{MHz})$ & $90678.0(11)$ & \\
\hline$E_{J}^{*}(\mathrm{MHz})$ & $0.40917(10)$ & \\
\hline$E_{K}^{*}(\mathrm{MHz})$ & $-3.085(82)$ & \\
\hline$E_{2}^{*}(\mathrm{MHz})$ & $0.18311(20)$ & \\
\hline$E_{J J}^{*}(\mathrm{kHz})$ & $-0.001921(51)$ & \\
\hline$E_{2 J}^{*}(\mathrm{kHz})$ & $-0.00150(10)$ & \\
\hline$F_{y z}(\mathrm{MHz})$ & $36.7781(57)$ & \\
\hline$F_{x y}(\mathrm{MHz})$ & $-0.1715(42)$ & \\
\hline$F_{y z K}(\mathrm{MHz})$ & $0.00583(15)$ & \\
\hline
\end{tabular}

a MP2/aug-cc-pVTZ calculations.

b Numbers in parentheses are two standard deviations in the same units as the last digit.

c Fixed to ab initio value.

\section{Conclusions}

The results of the current study provide a reliable basis for the detection of methoxymethanol in the ISM. The most stable conformation I was assigned and analyzed. However, low dipole moment value of the conformation I may be rather discouraging for astrophysical observations. Compared to the $\mathrm{CH}_{3} \mathrm{O}$ and $\mathrm{CH}_{2} \mathrm{OH}$ radicals, the dipole moment of the conformer I of methoxymethanol is lower by factor of 7.5 and 5.8 respectively. The intensity of rotational lines scales as a square of dipole moment. At a characteristic temperature of $10 \mathrm{~K}$, assuming equal abundance of the two radicals and $\mathrm{CH}_{3} \mathrm{OCH}_{2} \mathrm{OH}$, and taking the rotational partition functions into account one gets roughly relative intensities factors of 250 in case of $\mathrm{CH}_{3} \mathrm{O} / \mathrm{CH}_{3} \mathrm{OCH}_{2} \mathrm{OH}$, and 73 in case of $\mathrm{CH}_{2} \mathrm{OH} / \mathrm{CH}_{3} \mathrm{OCH}_{2} \mathrm{OH}$. The hydroxymethyl radical has not yet been detected in the ISM. The $\mathrm{CH}_{3} \mathrm{O}$ radical was detected in different cold sources ${ }^{6,33}$ but the mechanisms of its formation are not clearly understood. It is generally believed that in cold prestellar cores, methanol is formed on grains via successive CO hydrogenation, with $\mathrm{CH}_{3} \mathrm{O}$ as one of the intermediates of such reaction. The experimental simulations at $10 \mathrm{~K}$ confirm such possibility of the methoxy radical formation. ${ }^{5}$ In addition, these experiments also suggest that the dominant formation pathway of $\mathrm{CH}_{2} \mathrm{OH}$ is the hydrogen abstraction of $\mathrm{CH}_{3} \mathrm{OH}$. Theoretical calculations show that at low temperature $\mathrm{CH}_{3} \mathrm{O}$ radical may isomerise into $\mathrm{CH}_{2} \mathrm{OH}^{34}$ providing a possibility of the methoxy radical sink. If such isomerization is indeed effective in the ISM conditions, then subsequent recombination of $\mathrm{CH}_{3} \mathrm{O}$ and $\mathrm{CH}_{2} \mathrm{OH}$ radicals would lead to higher abundance of methoxymethanol making its detection more probable. However, at present, there are no reliable experimental data on isomerization of $\mathrm{CH}_{3} \mathrm{O}$ at low temperatures, and on the $\mathrm{CH}_{3} \mathrm{O}+\mathrm{CH}_{2} \mathrm{OH}$ reaction rates. In this view, the results of the present study would also be useful for exploring these two problems by means of high resolution rotational spectroscopy.

\section{Acknowledgements}

This work was supported by the French Programme National "Physique et Chimie du Milieu Interstellaire", and by ANR-13-BS05-0008 IMOLABS of the French Agence Nationale de la Recherche.

\section{References}

1 J. A. Ball, C. A. Gottlieb, A. E. Lilley and H. E. Radford, The Astrophysical Journal, 1970, 162, L203. 
2 D. Gerlich and M. Smith, Physica Scripta, 2005, 73, C25.

3 W. D. Geppert, M. Hamberg, R. D. Thomas, F. Österdahl, F. Hellberg, V. Zhaunerchyk, A. Ehlerding, T. J. Millar, H. Roberts, J. Semaniak et al., Faraday Discussions, 2006, 133, 177-190.

4 C. Pirim and L. Krim, Chemical Physics, 2011, 380, 67-76.

5 K.-J. Chuang, G. Fedoseev, S. Ioppolo, E. F. van Dishoeck and H. Linnartz, Monthly Notices of the Royal Astronomical Society, 2015, 455, 1702-1712.

6 J. Cernicharo, N. Marcelino, E. Roueff, M. Gerin, A. Jiménez-Escobar and G. M. Muñoz Caro, The Astrophysical Journal Letters, 2012, 759, L43.

7 C. Bermudez, S. Bailleux and J. Cernicharo, Astronomy \& Astrophysics, 2017, 598, A9.

8 J. M. Hollis, F. J. Lovas, P. R. Jewell and L. Coudert, The Astrophysical Journal Letters, 2002,571, L59.

9 C. Walsh, T. J. Millar, H. Nomura, E. Herbst, S. W. Weaver, Y. Aikawa, J. C. Laas and A. I. Vasyunin, Astronomy \& Astrophysics, 2014, 563, A33.

10 T. D. Harris, D. H. Lee, M. Q. Blumberg and C. R. Arumainayagam, The Journal of Physical Chemistry, 1995, 99, 9530-9535.

11 M. C. Boyer, M. D. Boamah, K. K. Sullivan, C. R. Arumainayagam, M. Bazin, A. D. Bass and L. Sanche, The Journal of Physical Chemistry C, 2014, 118, 22592-22600.

12 M. C. Boyer, N. Rivas, A. A. Tran, C. A. Verish and C. R. Arumainayagam, Surface Science, 2016, 652, 26-32.

13 K. K. Sullivan, M. D. Boamah, K. E. Shulenberger, S. Chapman, K. E. Atkinson, M. C. Boyer and C. R. Arumainayagam, Monthly Notices of the Royal Astronomical Society, 2016, 460, 664-672.

14 B. M. Hays and S. L. Widicus Weaver, The Journal of Physical Chemistry A, 2013, 117, 7142-7148.

15 B. M. Hays, N. Wehres, B. A. DePrince, A. A. Roy, J. C. Laas and S. L. W. Weaver, Chemical Physics Letters, 2015, 630, 18-26.

16 M. Priede, M. Kazak, T. Kalnins, K. Shubin and E. Suna, The Journal of Organic Chemistry, 2014, 79, $3715-3724$.

17 O. Zakharenko, R. A. Motiyenko, L. Margulès and T. R. Huet, Journal of Molecular Spectroscopy, 2015, 317, 41-46.

18 M. J. Frisch, G. W. Trucks, H. B. Schlegel, G. E. Scuseria, M. A. Robb, J. R. Cheeseman, G. Scalmani, V. Barone, B. Mennucci, G. A. Petersson et al., Gaussian 09, revision D.01, 2009.

19 C. Møller and M. S. Plesset, Physical Review, 1934, 46, 618.

20 T. H. Dunning Jr, The Journal of Chemical Physics, 1989, 90, 1007-1023.

21 A. D. Becke, Physical Review A, 1988, 38, 3098.

22 C. Lee, W. Yang and R. G. Parr, Physical Review B, 1988, 37, 785.

23 Y. Zhao and D. G. Truhlar, Theoretical Chemistry Accounts: Theory, Computation, and Modeling (Theoretica Chimica Acta), 2008, 120, 215-241.

24 O. Zakharenko, R. A. Motiyenko, J.-R. Aviles Moreno, A. Jabri, I. Kleiner and T. R. Huet, The Journal of Chemical Physics, 2016, 144, 024303.

25 O. Zakharenko, R. A. Motiyenko, J.-R. Aviles Moreno and T. R. Huet, The Journal of Physical Chemistry A, 2017, 121, 6420-6428.

26 Y. Niide and M. Hayashi, Journal of Molecular Spectroscopy, 2004, 223, 152-165. 
27 V. Ilyushin, A. Kryvda and E. Alekseev, Journal of Molecular Spectroscopy, 2009, 255, 32-38.

28 L. Kolesniková, J. Alonso, C. Bermúdez, E. Alonso, B. Tercero, J. Cernicharo and J.-C. Guillemin, Astronomy E Astrophysics, 2016, 591, A75.

29 H. Hartwig and H. Dreizler, Zeitschrift für Naturforschung A, 1996, 51, 923-932.

30 H. M. Pickett, The Journal of Chemical Physics, 1972, 56, 1715-1723.

31 L. Margulès, B. A. McGuire, M. L. Senent, R. A. Motiyenko, A. Remijan and J. C. Guillemin, Astronomy E Astrophysics, 2017, 601, A50.

32 R. A. Motiyenko, L. Margulès and J.-C. Guillemin, 70th International Symposium on Molecular Spectroscopy, 2015, RI02.

33 A. Bacmann and A. Faure, Astronomy \& Astrophysics, 2016, 587, A130.

34 T. Wang and J. H. Bowie, Organic ES Biomolecular Chemistry, 2012, 10, 3219-3228. 